\title{
Ceramides and sphingosine-1-phosphate as potential markers in diagnosis of ischaemic stroke
}

\author{
Anna Fiedorowicz ${ }^{2}$, Anna Kozak-Sykała ${ }^{1}$, Łukasz Bobak ${ }^{3}$, Wojciech Kałas ${ }^{4}$, Leon Strządała ${ }^{2}$ \\ ${ }^{1}$ Neurology and Stroke Department of Independent Public Healthcare Centre, Jankowski Regional Hospital in Przeworsk, Poland \\ ${ }^{2}$ Institute of Immunology and Experimental Therapy, Polish Academy of Sciences, Wroclaw, Poland
}

${ }^{3}$ Department of Animal Products Technology and Quality Management, Faculty of Food Sciences, Wroclaw University of Environmen-

tal and Life Sciences, Wroclaw, Poland

${ }^{4}$ Jan Dlugosz University in Czestochowa, Poland

\begin{abstract}
Background. Brain imaging in stroke diagnostics is a powerful tool, but one that can fail in more challenging cases, and one that is not particularly useful in identifying transient ischaemic attacks (TIAs). Thus, new reliable blood biomarkers of cerebral ischaemia are constantly sought.

Objective. We studied the potential usefulness of sphingolipids (SFs) as biomarkers of acute ischaemic stroke and TIA.

Material and methods. Levels of individual ceramide species and sphingosine-1-phosphate (Sph-1-P) in blood serum of patients with acute ischaemic stroke, TIA, and age-matched neurological patients without cerebral ischaemia, were assessed by tandem mass spectrometry liquid chromatography (LC- MS / MS).

Results. Wefound significant increases of several sphingolipid levels, with particularly strong elevations of Cer-C20:0 in patients with acute stroke. Cer-C24:1 was the only ceramide species to decrease as a result of acute stroke. Moreover, its levels inversely correlated with the number of days after stroke onset, suggesting that Cer-C24:1 is an independent parameter related to the course of stroke. To increase the sensitivity of sphingolipid-based tests in stroke diagnostics, we calculated the values of ratios of Sph-1-P / individual ceramide species and Cer-C24:1 individual ceramide species. Wefound several ratios significantly changed in stroke patients. Two ratios, Sph-1-P / Cer-C24:1 and Cer-C24:0 / Cer-C24:1, presented especially strong increments in patients with acute stroke. Moreover, Sph-1-P / Cer-C24:1 values were augmented in TIA patients.

Conclusion. Serum SFs could be good candidates to be ischaemic stroke biomarkers. We have identified two SF ratios, Sph-1-P / Cer-C24:1 and Cer-C24:0 / Cer-C24:1, with strong diagnostic potential in ischaemic stroke. Wefound Sph-1-P / Cer-C24:1 ratio to be possibly useful in TIAdiagnostics, also in the long term after ischaemic incidence.
\end{abstract}

Key words: ischaemic stroke marker, biomarker, brain, ceramide, mass spectrometry, observational clinical studies, sphingolipid, sphingosine-1-phosphate (Sph-1-P), transient ischaemic attack (TIA)

(Neurol Neurochir Pol 2019; 53 (6): 484-491)

\section{Introduction}

A diagnosis of ischaemic stroke is entirely based on neurological outcomes and brain imaging [1]. Although neuroimaging is a powerful technique for identifying brain ischaemia, it has many limitations. Using computer tomography (CT), the identification of an early ischaemic incident (i.e. less than three hours from onset) can be challenging and frequently requires the use of brain contrast agents [1]. In more difficult cases, only by the employment of a combination of several neuroimaging techniques can a full picture of the patient's condition be obtained. This is however not always

Address for correspondence: Anna Kozak-Sykała, Neurology and Stroke Department of Independent Public Healthcare Centre, Jankowski Regional Hospital in Przeworsk, Szpitalna 16, 37-200 Przeworsk, Poland; e-mail: annak@spzoz-przeworsk.pl 
feasible in a hospital setting. Moreover, the conditions of many patients substantially hinder the use of magnetic resonance imaging (MRI), which enables the recognition of early ischaemic stroke.

Another important issue is the diagnosis of TIA, which is mainly identified based on the ischaemic stroke-like symptoms, and is often challenging to confirm using CT / MR scans. Blood biomarkers will certainly not replace imaging studies in stroke diagnostics, but they do give several advantages in relation to CT / MR scans. Firstly, they can be helpful in the identification of early acute ischaemic stroke and TIA. The extended brain image-based diagnostics often require the patient being transported to a more specialist institution, which is not necessary when blood markers are analysed. The ideal stroke biomarker would allow physicians to follow the stroke course, showing the time of reperfusion, and helping to predict the outcome. The thromboembolic occlusion of the brain vessel leads to compromising of the blood-brain barrier (BBB), and enables blood elements to pass into brain parenchyma [2], as well as the leakage of inflammatory mediators and components of the damaged neuronal cells into the circulation. Many brain-derived and coagulation-related factors have been analysed to find the most useful ischaemic stroke biomarkers [3]. These factors have included IL-6, TNFa, GFAP, S100 $\beta$, D-dimers or thrombin-anti-thrombin complex, and several others. Although promising initial results were obtained, their clinical accuracy was insufficient.

In our study, we proposed ceramide (Cer) and sphingosine-1-phosphate (Sph-1-P) as potential ischaemic stroke biomarkers, the biological features of which could allow several stroke-related processes to be tracked in the future. Cer is composed of sphingoid base, at large d18:1, linked to fatty acid chain. Acyl chain in Cer varies greatly in length and bond saturation $[4,5]$. The most common pathways of Cer generation are de novo synthesis and sphingomyelin hydrolysis. Over-activations of both pathways have been reported in animal models of cerebral ischaemia [6-8]. The Cer form diversity is created by several Cer synthases [9]. Little is known about the specific functions of individual Cer species since they have been predominantly studied in regards to cell membrane structure [10]. In contrast to Cer, Sph-1-P exerts biological functions by binding to its receptors, although alternative pathways have also been reported [11]. Cerebral ischaemia alters both Cer and Sph-1-P concentrations in affected tissues. Cer accumulates in the brain shortly after ischaemia and has generally been considered to be a mediator of neurodegeneration [12-14]. Cer created in the brain can potentially get through the impaired BBB to the circulation, changing Cer profiles in blood serum / plasma. In contrast to Cer, the amounts of Sph-1-P tend to decrease in ischaemic stroke [15]. Sph-1-P reaches particularly high concentrations in blood, where it is mainly produced by red blood cells and endothelial cells [16]. However, during clot formation, Sph-1-P stored in high amounts in platelets is released extracellularly, and increases substantially the Sph-1-P content in blood serum [17]. It is known that Sph-1- $P$ augments the level of plasminogen activator inhibitor-1 (PAI-1) [18], and thus can constrain fibrinolysis. This may impede spontaneous recanalisation in stroke patients, and produce more severe brain insults.

Although there is a great deal of data on Cer and Sph-1-P changes in post-stroke animal brains, almost nothing is known about stroke-induced sphingolipid alterations in human blood. The main goal of this study was to establish whether Cer and Sph-1-P levels are altered in the serum of patients after ischaemic brain stroke and TIA, and whether they could in future be considered as potential ischaemic stroke biomarkers. Moreover, in calculating the ratios of selected SFs, we have attempted to find a method of SF data presentation which would make the obtained results more informative for their potential use in stroke diagnostics.

\section{Material and methods}

\section{Baseline characteristics of the patients}

The overall characteristics of the patients are set out in Table 1 . 103 patients were enrolled in the study. 42 were diagnosed with ischaemic stroke on the basis of specific symptoms and CT and MR scans. The grade of neurological dysfunction was assessed at admission using the NIH Stroke Scale (NIHSS). TIA was

Table 1. Baseline characteristics of patients

\begin{tabular}{|c|c|c|c|}
\hline & $\begin{array}{l}\text { Controls } \\
(n=34)\end{array}$ & $\begin{array}{c}\text { Transient ischaemic } \\
\text { attack } \\
(\mathbf{n}=\mathbf{2 7})\end{array}$ & $\begin{array}{c}\text { Acute cerebral } \\
\text { ischaemia } \\
(n=42)\end{array}$ \\
\hline Females (\%) & 60 & 63 & 67 \\
\hline Males (\%) & 40 & 37 & 33 \\
\hline Age - median (Q1; Q3) & $69.5(62 ; 78.5)$ & $71(62.5 ; 78.5)$ & $76(71.5 ; 82)$ \\
\hline Patients with diabetes (\%) & 15 & 15 & 39 \\
\hline Patients with hypertriglyceridemia (\%) & 21 & 21 & 21 \\
\hline Patients with hypercholesterolemia (\%) & 24 & 27 & 18 \\
\hline Patients with renal disease (\%) & 15 & 15 & 36 \\
\hline
\end{tabular}


identified in 27 patients on the basis of ischaemic stroke-like symptoms that had disappeared within 24 hours of onset and did not exhibit changes in image scans. Among the 34 participants in the control group were age-matched patients of the Neurological Department suffering from various neurological disorders but who had not experienced an ischaemic stroke / TIA. These neurological diseases included sciatica, polyneuropathy, spinal disc herniation, epilepsy, Parkinson's disease, cervicobrachial syndrome, cerebrovascular disease, and facial nerve disorder. The common morphological and biochemical parameters were measured in all persons enrolled in the study. Patients were classified as those with hypercholesterolemia and hypertriglyceridemia by assessing the levels of total cholesterol and triglycerides in the blood, respectively. The cut off was established at $150 \mathrm{mg}$ / dL for triglyceride level and $190 \mathrm{mg} \%$ for total cholesterol level. Subjects with diabetes were identified based on the medical history and a glucose tolerance test. Renal disease was diagnosed by assessing the levels and clearance of creatinine (above $1.17 \mathrm{mg} / \mathrm{dL}$ ) as well as an estimated glomerular filtration rate (eGFR) of less than $60 \mathrm{~mL} / \mathrm{min}$.

Blood samples were collected after receiving informed consent approved by the local ethics board (The Regional Medical Chamber in Krakow), according to the Declaration of Helsinki. Blood was drawn 2-21 days after ischaemic stroke onset, and 2-7 days after TIA.

\section{Lipid isolation}

Lipids from blood serum were extracted on the basis of the method described by Folch et al. [19]. Before extraction, serum samples were spiked with internal standards, d17:0-Cer or d17:1-Sph-1-P AVANTI (Polar Lipids Inc., Alabaster, AL, USA). $1 \mathrm{~mL}$ of a chloroform / methanol mixture $(2: 1, \mathrm{v} / \mathrm{v})$ and $1 \mathrm{ml}$ of $0.15 \mathrm{M} \mathrm{NaCl}$ solutions were added to the serum sample. Then the mixture was shaken vigorously and placed into an ultrasonic bath for $10 \mathrm{~min}$. Phases were separated by centrifugation (1,900 xg, $3 \mathrm{~min}$ ), and the chloroform layer was evaporated in centrifugal vacuum concentrators at $60^{\circ} \mathrm{C}$ (Labconco, Kansas City, MO, USA). Dry residue was resuspended in 1,000 $\mu \mathrm{l}$ phase B (acetonitrile / 2-propanol (60:40, $\mathrm{v} / \mathrm{v}$ ) containing $0.2 \%$ formic acid) and filtered with a 0.22 $\mu \mathrm{m}$ PTFE syringe filter.

\section{Sphingolipid analysis}

The assay was conducted using the technique of Kasumov et al. (2006) [20] with slight modifications. Pure standards of Cers (CerC14:0, CerC16:0, CerC18:0, CerC20:0, CerC24:0 and CerC24:1) and Sph-1-P were obtained from AVANTI Polar Lipids. Extracted samples were injected into Agilent 1200 HPLC and separated through a ZORBAX Eclipse Plus C18 column $(2.1 \times 50 \mathrm{~mm}, 3,5 \mu \mathrm{m}$, (Agilent Technologies, Palo Alto, CA, USA). The Cers were resolved using a gradient starting from $50 \%$ mobile phase A (water containing $0.2 \%$ formic acid) at a flow rate of $0.3 \mathrm{~mL} / \mathrm{min}$ for $1 \mathrm{~min}$, to $100 \%$ mobile phase B [acetonitrile / 2-propanol (60:40, v / v) containing
$0.2 \%$ formic acid] over $3 \mathrm{~min}$ at a linear gradient, and then with $100 \%$ B for $12 \mathrm{~min}$. The column was then equilibrated with $50 \%$ mobile phase B. The HPLC column effluent was introduced onto a triple quadrupole mass spectrometer 6410 (Agilent Technologies, Palo Alto, CA, USA) and analysed using electrospray ionisation in the positive ion mode with multiple reaction monitoring. Data was acquired using MassHunter acquisition software (Agilent Technologies revision B.04.00, Palo Alto, CA, USA).

\section{Statistical analysis}

A Kruskal-Wallis rank test with a post hoc test was used to calculate multiple comparisons of the studied groups of patients. The statistical relations between two variables were found using Spearman's rank coefficient. The results were considered statistically significant when $\mathrm{p}<0.05$. All calculations were performed using STATISTICA v.13.1 (StatSoft. Inc., USA).

\section{Results}

The levels of Cer-C14:0, Cer-C24:0, Cer-C20:0 and Sph-1-P are augmented, and Cer-C24:1 decreased, in serum of patients with acute ischaemic stroke.

Multiple significant changes in SF plasma concentrations were found in patients with acute ischaemic stroke compared to non-stroke participants.

The acute stroke-induced changes in levels of several Cer species are shown in Figs. 1A, B. Significantly augmented concentrations of Cer-C14:0 ( $p=0.049)$ and Cer-C24:0 $(\mathrm{p}=0.023)$ were found in patients after acute stroke when compared to the group of patients without stroke. Particularly high increments were noted for Cer-C20:0 ( $p<0.001)$, the content of which was almost doubled in people with brain infarction (Fig. 1A). In contrast, the levels of Cer-C24:1 $(p=0.013)$ were significantly decreased in subjects with acute stoke. Cer-C24:1 was the only studied SF of which the concentrations were lower in the stroke population than in the group without stroke (Fig. 1B). Moreover, the levels of Cer-C24:1 showed a significant inverse correlation with the time after stroke onset $(R=-0.40, p=0.012)$, but not with any other analysed parameters (Fig. 1C). This suggests that Cer-C24:1 is an independent parameter related to the course of stroke. The concentrations of Cer-C16:0 (not shown) and Cer-C18:0 (Fig. 1C) positively correlated with NIHSS (R=0.46, $\mathrm{p}=0.003$; $\mathrm{R}=0.39, \mathrm{p}=0.011$, respectively), although their levels were not significantly altered in acute stroke.

On the other hand, both SF species showed positive relations with the presence of diabetes (Cer-C16:0 $-\mathrm{R}=0.38$, $\mathrm{p}=0.014 ;$ Cer-C18:0 $-\mathrm{R}=0.46, \mathrm{p}=0.003)($ Cer-C16:0 - not shown; Cer-C18:0 - Fig. 1C), but diabetes by itself was a predictive factor for poor patient outcomes after stroke $(\mathrm{R}=0.53, \mathrm{p}<0.001)$. An acute stroke led also to a significant elevation of Sph-1-P in blood serum $(\mathrm{p}=0.020)$ (Fig.1A), although levels of Sph-1-P did not correlate either with time 


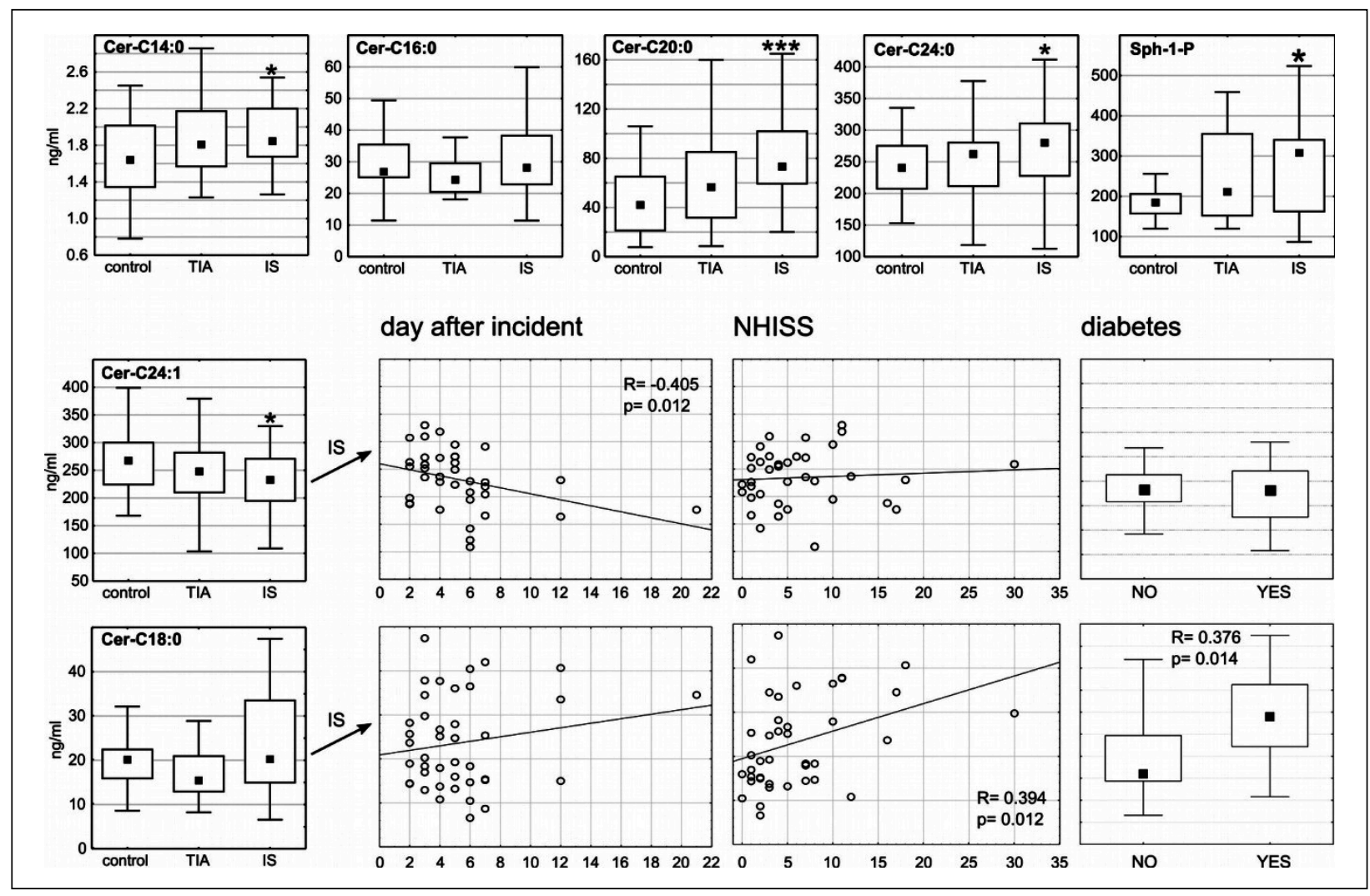

Figure 1. Levels of individual Cer species and Sph-1-P in blood serum (A, B) and their selected correlations with the number of days after ischaemic stroke onset, NIHSS, and diabetes (C). The concentrations of studied SF species are presented as median (Q1-Q3) values. Selected Spearman's rank correlations are shown as scatter graphs (number of days after ischaemic stroke onset and NIHSS). Correlations between levels of selected SFs and diabetes are presented on plots of SF concentration median (Q1-Q3) values in ischaemic patients with and without diabetes

${ }^{*} \mathrm{p}<0.05$

***p $<0.001$ versus controls

TIA — transient ischaemic attack; IS - ischaemic stroke

afterstroke onset or with NIHSS. The serum concentrations of those SFs which were altered in acute ischaemic stroke were also slightly changed in TIA patients, although not sufficiently to reach statistical significance.

All Spearman's rank correlation coefficients, calculated between levels of individual Cer species as well as Sph-1-P and number of days after stroke, and NIHSS, are set out in Table 2. Selected scatter graphs are shown in Figure 1C.

The serum ratios of Sph-1-P / C24:1 and C24:0 / Cer-C24:1 are strongly increased in patients with acute ischaemic stroke, and Sph-1-P / C24:1 ratios are raised after TIA.

To strengthen the accuracy of the SF system in potential stroke diagnostics, we calculated ratios:

1) Sph-1-P / individual Cer species and 2) individual Cer species / Cer-C24:1. The first ratio was chosen so as to integrate factors reflecting clot formation (Sph-1-P) and BBB damage (Cer). The second ratio was evaluated having regards to the fact that Cer-C24:1 was the only studied Cer species exhibiting a significant decrease in stroke patients. Most studied ratios of
Sph-1-P / individual Cer species were significantly changed in patients with acute stoke compared to non-stroke controls, although most of the alterations were quite modest. Among calculated Sph-1-P / Cer ratios, Sph-1-P / Cer-C24:1 was found to be highly augmented in patients with acute stroke ( $\mathrm{p}<0.001$ ) (Fig. 2). Moreover, Sph-1-P / Cer-C24:1 was also significantly increased in people after TIA compared to non-ischaemic controls ( $\mathrm{p}=0.008)$ (Fig. 2).

Similarly, most of the studied ratios of individual Cer species / Cer-C24:1 were significantly higher in patients who had experienced an acute ischaemic stroke compared to the non-stroke group, but especially high values were found for Cer-C24:0 / Cer-C24:1 (p < 0.001) (Fig. 2). Interestingly, choosing a value of $\mathrm{Q} 3$ of control as a reference, we found that over $80 \%$ of patients with acute stroke, and $60 \%$ of patients after TIA, presented Sph-1-P / Cer-C24:1 values above Q3 of control (Fig. 2). Similarly, approximately $80 \%$ of stroke patients showed Cer-C24:0 / Cer-C24:1 ratios higher than value of Q3 of control (Fig. 2). 


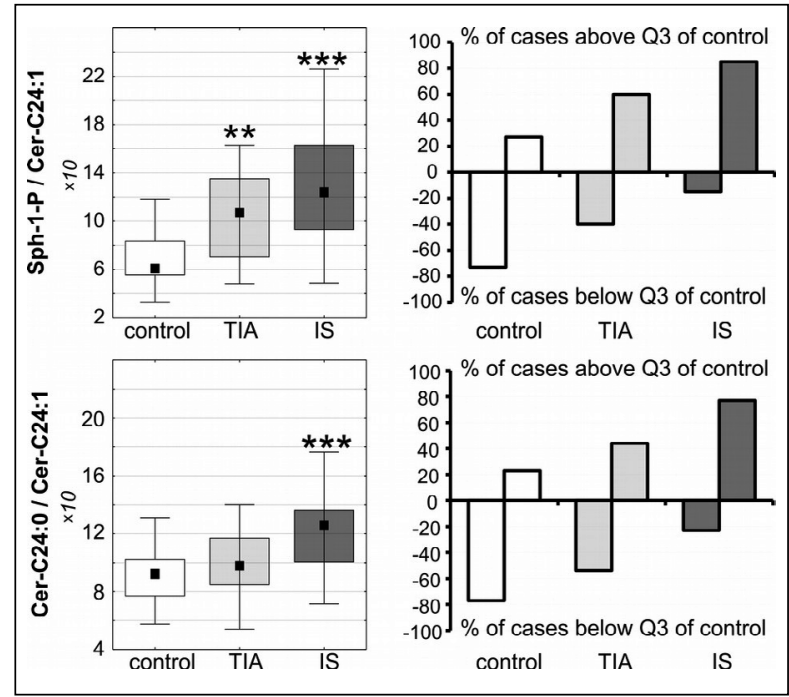

Figure 2. Selected ratios of blood serum SFs. Values of SF ratios are presented as median (Q1-Q3) values. Percentage of patients presented selected SF ratio values in relation to Q3 of control as a reference is shown as \% of cases above and below Q3 of control ${ }^{* *} p<0.01, * * * p<0.001$ versus control

TIA — transient ischaemic attack; IS - ischaemic stroke

The correlations between levels of Cer, Sph-1-P as well as chosen SF ratios and selected clinical parameters in patients with acute ischaemic stroke.

Several clinical conditions, such as diabetes, hypercholesterolemia and hypertriglyceridemia as well as renal disease, which presented in subpopulations of patients with acute ischaemic stroke, were analysed in order to assess their potential correlations with studied SFs and chosen SF ratios (Tab. 2). No significant correlations between studied SFs or selected SF ratios and hypercholesterolemia or renal disease were found in people after acute stroke. In turn, the presence of hypertriglyceridemia exhibited weak positive correlations only with Cer-C18:0 concentrations and Cer-C18:0 / Cer-C24:1 ratios in stroke patients. Furthermore, levels of Cer-C16:0 and Cer-C18:0, as well as their ratios: Cer-C16:0 / Cer-C24:1, Cer-C18:0 / Cer-C24:1, Sph-1-P / Cer-C16:0, Sph-1-P / Cer-C18:0 significantly correlated with the presence of diabetes in stroke patients. In addition, the overall group of participants ( $n=97-99)$, including people with acute stroke, TIA and non-stroke controls was analysed in order to find the potential correlations of studied SFs with age and sex (not shown). Among the studied parameters, only concentrations of Cer-C14:0 showed faint correlations with age. No other statistically significant correlations with age and sex were reported, which excludes the possibility that the observed changes in SF levels were related to ageing or may have been influenced by the sex of the patient.

\section{Discussion}

Despite the SF system having been tested for potential diagnostic utility in several diseases [21-23], the only study showing dramatic changes of numerous Cer species in CSF in stroke was focused on subarachnoid haemorrhages [24]. No conclusive research has been performed in ischaemic stroke, even though Cer is considered to be an important factor contributing to atherosclerotic plaque formation [25]. Recently, Laaksonen et al. (2016) [26] proved that several Cer species, and particularly their specific ratios, were strong predictors of death risk in cardiovascular disease, independent of other lipid markers. In our study, we found significantly augmented serum content of Cer-C14:0, Cer-C24:0 and particularly strong elevation of Cer-C20:0 in patients after ischaemic stroke. It should be noted that concentrations of all these forms of Cer were increased in a heterogeneous group of stroke patients, covering a range of more than a week after stroke onset. This suggests that the observed changes may be sustained for a longer period of time, which makes these Cer species good candidates to be stroke biomarkers. Surprisingly, we noted that levels of Cer-24:1, as the only Cer species, were significantly diminished in stroke patients. Moreover, Cer-C24:1 was the only studied SF, which correlated inversely with the number of days after stroke onset. Thus, its levels were decreased over time in the course of a stroke. The altered Cer species did not correlate with cholesterol levels, which clearly shows that serum concentrations of these Cer forms are not elevated as a consequence of higher content of their carriers.

This makes Cer an independent indicator of ischaemic stroke. Furthermore, we observed positive correlations of Cer-C18:0 and Cer-C16:0 with NIHSS, indicating worse outcomes of patients with high levels of these Cers. However, we found that serum concentrations of both Cers correlated with the presence of diabetes, which is associated with a poor prognosis for stroke

patients [27]. Therefore, Cer-C18:0 and Cer-C16:0 cannot be taken into account as independent prognostic markers in ischaemic stroke.

We have shown that, along with Cer, Sph-1-P serum concentrations were significantly augmented as a result of cerebral infarction. Similarly to Cer, Sph-1-P was found to have accumulated in ischaemia-insulted brain regions, but as late as seven days after stroke onset [28]. On the contrary, early after focal cerebral ischaemia, Sph-1-P was depleted from damaged brain tissue. During thrombosis, circulating Sph-1-P is massively released from platelets, which increases substantially its blood content [17]. Sph-1-P has been shown to promote clotting [18], which would be an undesirable effect for patients with ischaemic stroke.

Although no significant alterations in studied SFs were found in patients after TIA, we observed minor fluctuations of these lipids directed similarly to changes of respective species in acute stroke. This suggests that TIA also influences Cer and Sph-1-P serum profiles, but to a much smaller extent than acute ischaemic stroke. Moreover, since TIA does not produce an extensive brain insult, TIA-evoked SF alterations are less abundant and a short period of time is sufficient to restore their blood balance. 
Table 2. Spearman's rank correlation coefficients between serum levels of individual Cer species, Sph-1-P as well as Sph-1-P / individual Cer species and individual Cer species / Cer-C24:1 ratios, and selected patient parameters in the ischaemic stroke group

\begin{tabular}{|c|c|c|c|c|c|c|}
\hline & Day after admission & NIHSS & Diabetes & HCHOL & HTG & Renal disease \\
\hline Cer-C14:0 (ng / ml) & -0.04 & 0.08 & -0.03 & -0.22 & -0.22 & 0.09 \\
\hline Cer-C16:0 (ng / ml) & 0.04 & $0.46 * *$ & $0.38^{*}$ & -0.1 & 0.22 & -0.05 \\
\hline Cer-C18:0 (ng / ml) & 0.02 & $0.39 *$ & $0.46^{* *}$ & -0.18 & $0.32^{*}$ & -0.1 \\
\hline Cer-C20:0 (ng / ml) & -0.29 & -0.04 & -0.19 & -0.03 & -0.03 & -0.05 \\
\hline Cer-C24:0 (ng / ml) & -0.02 & -0.16 & -0.02 & -0.18 & -0.25 & -0.12 \\
\hline Cer-C24:1 (ng / ml) & $-0.40 *$ & 0.14 & -0.1 & -0.22 & -0.15 & -0.01 \\
\hline Sph-1-P (ng / ml) & -0.25 & 0.01 & 0.1 & 0.13 & 0.06 & -0.08 \\
\hline Cer-C14:0 / Cer-C24:1 & 0.24 & -0.06 & 0.14 & 0.02 & 0.06 & 0.19 \\
\hline Cer-C16:0 / Cer-C24:1 & 0.29 & $0.45^{* *}$ & $0.47^{* *}$ & 0.06 & 0.27 & -0.14 \\
\hline Cer-C18:0 / Cer-C24:1 & 0.16 & $0.33^{*}$ & $0.42^{* *}$ & 0.00 & $0.37^{*}$ & -0.07 \\
\hline Cer-C20:0 / Cer-C24:1 & 0.18 & 0.01 & -0.07 & -0.02 & -0.02 & -0.02 \\
\hline Cer-C24:0 / Cer-C24:1 & 0.31 & -0.27 & 0.02 & 0.00 & -0.19 & 0.14 \\
\hline Sph-1-P / Cer-C14:0 & -0.28 & -0.04 & 0.07 & 0.20 & 0.10 & -0.04 \\
\hline Sph-1-P / Cer-C16:0 & -0.25 & $-0.31^{*}$ & -0.15 & 0.12 & -0.14 & 0.06 \\
\hline Sph-1-P / Cer-C18:0 & -0.18 & $-0.35^{*}$ & -0.27 & 0.20 & -0.26 & 0.02 \\
\hline Sph-1-P / Cer-C20:0 & -0.07 & -0.06 & 0.17 & 0.27 & 0.13 & 0.14 \\
\hline Sph-1-P / Cer-C24:0 & -0.26 & 0.09 & 0.20 & 0.17 & 0.14 & -0.01 \\
\hline Sph-1-P / Cer-C24:1 & -0.12 & -0.14 & 0.17 & 0.20 & 0.05 & 0.03 \\
\hline
\end{tabular}

${ }^{*} p<0.05,{ }^{* * *} p<0.001$ versus control

$\mathrm{HCHOL}$ - hypercholesterolemia; HTG - hypertriglyceridemia

In order to strengthen the sensitivity of our measurements, we calculated numerous SF ratios, seeking those that were highly altered in ischaemic stroke / TIA. We selected two ratios which could potentially be applied in stroke diagnostics: Cer-C24:0 / Cer-C24:1 was significantly higher in patients with cerebral infarction. A second ratio, Sph-1-P / Cer-C24:1, was highly augmented not only in stroke patients, but also in people who had experienced TIA, which suggests its potential utility in TIA diagnostics. It should be noted that increments in the abovementioned SF ratios in stroke patients had greater statistical power than the changes in levels of individual SF species. Thus, we suggest that in further studies toward elaborating SF-based diagnostic tests, the use of SF ratios is highly desirable.

An important issue in studies on humans is the selection of control groups. In our study, the control group consisted of patients with various neurological disorders, but not ischaemic stroke / TIA. The control group matched the age and sex distributions of the stroke and TIA groups, as well as involving participants with similar comorbid diseases to ischemic patients. In that way, we could verify whether it is possible to distinguish patients with brain ischaemia from those with other causes of neurological dysfunction by using SF measurements.
The question arises as to what is the source of the increments in serum Cer levels? One possibility is associated with a recanalisation event. Full recanalisation is highly desirable within the first hours after a stroke [29]. However, unblocking an occluded vessel in the later stages of an ischaemic stroke can cause paradoxical effects such as a deterioration in the patient's condition [30]. Inflammatory processes in the infarcted brain areas lead to BBB damage [31], and subsequent reopening of the vessel results in a massive influx of immune cells into the brain parenchyma. On the other hand, it also allows for the leakage of factors produced in affected brain regions to the circulation. However, such events can be temporarily limited to the sub-recanalisation period. Thus, some Cer species accumulated in the brain in the early stages of stroke may be released to the blood rapidly during reperfusion, and stay invisible in analysis encompassing a range of up to 20 days after stroke onset.

It has been reported that Cer in an ischaemic brain is generated both due to de novo synthesis as well as sphingomyelin hydrolysis [6-8,32]. Although these observations seem inconsistent at first glance, they are not necessarily contradictory. Cer can be generated in the ischaemic brain over a longer time, first being produced early after stroke onset through SM 
hydrolysis, and then several days after the stroke incident in de novo synthesis pathway. Thus, accumulations of different Cer species in the insulted cerebral areas may be observed at different stroke stages. In blood, Cer has been shown to be a stimulator of tissue factor (TF) activity [33]. Because TF is essential in blood coagulation, the increased levels of Cer could contribute to the generation of a thromboembolism and predispose patients to recurrent strokes, which would be an additional aspect of Cer action.

It worth mentioning that the medications of patients used for the treatment of ischaemic stroke can influence the profile of plasma sphingolipids. Similarly, exposure to statins due to common chronic conditions affects plasma levels of a wide spectrum of lipids and sphingolipids [34]. Both factors should be considered in further studies performed on a larger cohort of patients.

\section{Conclusions}

We found serum SFs to be good candidates to be ischaemic stroke biomarkers. We identified two SF ratios, Sph-1-P / Cer-C24:1 and Cer-C24:0 / Cer-C24:1, with strong diagnostic potential in ischaemic stroke. We found Sph-1-P / Cer-C24:1 ratio to be possibly useful in TIA diagnostics, also over the long term after an ischaemic incident, something which is essential in anti-stroke preventive treatment.

Abbreviations: Cer - ceramide; Sph-1-P - sphingosine-1-phosphate; TIA - transient ischaemic attack; SF - sphingolipid; CT - computer tomography; IS - ischaemic stroke; MRI - magnetic resonance imaging; $B B B$ - blood brain barrier; GFAP - glial fibrillary acidic protein; NIHSS - NIH Stroke Scale; CSF - cerebrospinal fluid

\section{Acknowledgments}

This study was supported by Grant no. 101 from the Wroclaw Centre of Biotechnology, programme The Leading National Research Centre (KNOW) for years 2014-2018; IITD programme no. 3 / 2017.

\section{Conflicts of interest: None.}

\section{Contributions:}

$\mathrm{AF}$ - designed the study, isolated lipids, performed the statistical analysis, interpreted data, wrote the manuscript; AKS - designed the study, as MD had direct contact with patients and selected participants for the study, supervised the collection of human blood serum, read and critically revised the manuscript; LB - performed the sphingolipid analysis, wrote part of the manuscript; WK - performed the statistical analysis, interpreted data, read and critically revised the manuscript; LS - designed the study, interpreted data, read and critically revised the manuscript.

\section{References}

1. Nentwich LM. Diagnosis of Acute Ischemic Stoke. Emerg Med Clin North Am. 2016; 34(4): 837-859, doi: 10.1016 / j.emc.2016.06.008, indexed in Pubmed: 27741991.

2. Kassner A, Mandell DM, Mikulis DJ. Measuring permeability in acute ischemic stroke. Neuroimaging Clin N Am. 2011; 21(2): 315-325, doi: 10.1016 / j.nic.2011.01.004, indexed in Pubmed: 21640302.

3. Guo Y, Li P, Guo Q, et al. Pathophysiology and Biomarkers in Acute Ischemic Stroke - A Review. Tropical Journal of Pharmaceutical Research. 2014; 12(6): 1097, doi: 10.4314 / tjpr.v12i6.35.

4. Grösch S, Schiffmann S, Geisslinger G. Chain length-specific properties of ceramides. Prog Lipid Res. 2012; 51(1): 50-62, doi: 10.1016 / j.plipres.2011.11.001, indexed in Pubmed: 22133871.

5. Car H, Zendzian-Piotrowska M, Fiedorowicz A, et al. [The role of ceramides in selected brain pathologies: ischemia / hypoxia, Alzheimer disease]. Postepy Hig Med Dosw (Online). 2012; 66: 295-303, doi: 10.5604 / 17322693.999024, indexed in Pubmed: 22706115.

6. Yu J, Novgorodov SA, Chudakova D, et al. JNK3 signaling pathway activates ceramide synthase leading to mitochondrial dysfunction. J Biol Chem. 2007; 282(35): 25940-25949, doi: 10.1074 / jbc. M701812200, indexed in Pubmed: 17609208.

7. Gu L, Huang B, Shen W, et al. Early activation of nSMase2 / ceramide pathway in astrocytes is involved in ischemia-associated neuronal damage via inflammation in rat hippocampi. J Neuroinflammation. 2013; 10: 109, doi: 10.1186 / 1742-2094-10-109, indexed in Pubmed: 24007266.

8. Ohtani R, Tomimoto $\mathrm{H}$, Kondo $\mathrm{T}$, et al. Upregulation of ceramide and its regulating mechanism in a rat model of chronic cerebral ischemia. Brain Res. 2004; 1023(1): 31-40, doi: 10.1016 / j.brainres.2004.07.024, indexed in Pubmed: 15364016.

9. Mullen TD, Hannun YA, Obeid LM. Ceramide synthases at the centre of sphingolipid metabolism and biology. Biochem J. 2012; 441(3): 789802, doi: 10.1042 / BJ20111626, indexed in Pubmed: 22248339.

10. Goñi FM, Sot J, Alonso A. Biophysical properties of sphingosine, ceramides and other simple sphingolipids. Biochem Soc Trans. 2014; 42(5): 1401-1408, doi: 10.1042 / BST20140159, indexed in Pubmed: 25233422.

11. Hopson KP, Truelove J, Chun J, et al. S1P activates store-operated calcium entry via receptor- and non-receptor-mediated pathways in vascular smooth muscle cells. Am J Physiol Cell Physiol. 2011; 300(4): C919-C926, doi: 10.1152 / ajpcell.00350.2010, indexed in Pubmed: 21270296.

12. Tong M, de la Monte SM. Mechanisms of ceramide-mediated neurodegeneration. J Alzheimers Dis. 2009; 16(4): 705-714, doi: 10.3233 / JAD-2009-0983, indexed in Pubmed: 19387107.

13. Herr I, Martin-Villalba A, Kurz E, et al. FK506 prevents stroke-induced generation of ceramide and apoptosis signaling. Brain Research. 1999; 826(2): 210-219, doi: 10.1016 / s0006-8993(99)01288-3.

14. Nakane M, Kubota M, Nakagomi $T$, et al. Lethal forebrain ischemia stimulates sphingomyelin hydrolysis and ceramide generation in the gerbil hippocampus. Neuroscience Letters. 2000; 296(2-3): 89-92, doi: 10.1016 / s0304-3940(00)01655-4.

15. Kimura A, Ohmori T, Kashiwakura Y, et al. Antagonism of sphingosine 1-phosphate receptor-2 enhances migration of neural progenitor cells toward an area of brain. Stroke. 2008; 39(12): 3411-3417, doi: 10.1161 / STROKEAHA.108.514612, indexed in Pubmed: 18757288.

16. Thuy AV, Reimann CM, Hemdan NYA, et al. Sphingosine 1-phosphate in blood: function, metabolism, and fate. Cell Physiol Biochem. 2014; 
34(1): 158-171, doi: 10.1159 / 000362992 , indexed in Pubmed: 24977489.

17. Vito CDi, Hadi LA, Navone SE, et al. Platelet-derived sphingosine1-phosphate and inflammation: from basic mechanisms to clinical implications. Platelets. 2016; 27(5): 393-401, doi: 10.3109 / 09537104.2016.1144179, indexed in Pubmed: 26950429.

18. Iwaki S, Yamamura S, Asai M, et al. Posttranscriptional regulation of expression of plasminogen activator inhibitor type-1 by sphingosine 1-phosphate in HepG2 liver cells. Biochim Biophys Acta. 2012; 1819(11-12): 1132-1141, doi: 10.1016 / j.bbagrm.2012.07.001, indexed in Pubmed: 22819712.

19. Folch J, Lees M, Sloane Stanley GH. A simple method for the isolation and purification of total lipides from animal tissues. J Biol Chem. 1957; 226(1): 497-509, indexed in Pubmed: 13428781.

20. Kasumov T, Huang H, Chung YM, et al. Quantification of ceramide species in biological samples by liquid chromatography electrospray ionization tandem mass spectrometry. Anal Biochem. 2010; 401(1): 154-161, doi: 10.1016 / j.ab.2010.02.023, indexed in Pubmed: 20178771.

21. Haus JM, Kashyap SR, Kasumov T, et al. Plasma ceramides are elevated in obese subjects with type 2 diabetes and correlate with the severity of insulin resistance. Diabetes. 2009; 58(2): 337-343, doi: 10.2337 / db08-1228, indexed in Pubmed: 19008343.

22. Borodzicz S, Czarzasta K, Kuch M, et al. Sphingolipids in cardiovascular diseases and metabolic disorders. Lipids Health Dis. 2015; 14: 55, doi: 10.1186 / s12944-015-0053-y, indexed in Pubmed: 26076974.

23. Xing Yi, Tang Yi, Zhao L, et al. Plasma Ceramides and Neuropsychiatric Symptoms of Alzheimer's Disease. J Alzheimers Dis. 2016; 52(3): 1029-1035, doi: 10.3233 / JAD-151158, indexed in Pubmed: 27079712.

24. Testai FD, Hillmann M, Amin-Hanjani S, et al. Changes in the cerebrospinal fluid ceramide profile after subarachnoid hemorrhage. Stroke. 2012; 43(8): 2066-2070, doi: 10.1161 / STROKEAHA.112.650390, indexed in Pubmed: 22713492.

25. Edsfeldt A, Dunér P, Ståhlman M, et al. Sphingolipids Contribute to Human Atherosclerotic Plaque Inflammation. Arterioscler Thromb Vasc
Biol. 2016; 36(6): 1132-1140, doi: 10.1161 / ATVBAHA.116.305675, indexed in Pubmed: 27055903.

26. Laaksonen R, Ekroos K, Sysi-Aho M, et al. Plasma ceramides predict cardiovascular death in patients with stable coronary artery disease and acute coronary syndromes beyond LDL-cholesterol. European Heart Journal. 2016; 37(25): 1967-1976, doi: 10.1093 / eurheartj / ehw148.

27. Tuttolomondo A. Relationship between Diabetes and Ischemic Stroke: Analysis of Diabetes- Related Risk Factors for Stroke and of Specific Patterns of Stroke Associated with Diabetes Mellitus. Journal of Diabetes \& Metabolism. 2015; 06(05), doi: 10.4172 / 21556156.1000544.

28. Nielsen MMB, Lambertsen KL, Clausen BH, et al. Mass spectrometry imaging of biomarker lipids for phagocytosis and signalling during focal cerebral ischaemia. Sci Rep. 2016; 6: 39571, doi: 10.1038/ srep39571, indexed in Pubmed: 28004822.

29. Rha JH, Saver JL. The impact of recanalization on ischemic stroke outcome: a meta-analysis. Stroke. 2007; 38(3): 967-973, doi: 10.1161 / 01.STR.0000258112.14918.24, indexed in Pubmed: 17272772.

30. Lapchak P, Zhang A, John H. Translational Stroke Research. 2012, doi: 10.1007 / 978-1-4419-9530-8.

31. Anrather J, ladecola C. Inflammation and Stroke: An Overview. Neurotherapeutics. 2016; 13(4): 661-670, doi: 10.1007 / s13311-0160483-x, indexed in Pubmed: 27730544.

32. Brunkhorst R, Friedlaender F, Ferreirós N, et al. Alterations of the Ceramide Metabolism in the Peri-Infarct Cortex Are Independent of the Sphingomyelinase Pathway and Not Influenced by the Acid Sphingomyelinase Inhibitor Fluoxetine. Neural Plast. 2015, doi: 10.1155 / 2015 / 503079, indexed in Pubmed: 26605090.

33. Hirokawa M, Kitabayashi A, Kuroki J, et al. Induction of tissue factor production but not the upregulation of adhesion molecule expression by ceramide in human vascular endothelial cells. Tohoku J Exp Med. 2000; 191(3): 167-176, doi: 10.1620 / tjem.191.167, indexed in Pubmed: 10997557.

34. Ng TW, Ooi EM, E M, et al. Dose-dependent effects of rosuvastatin on the plasma sphingolipidome and phospholipidome in the metabolic syndrome. J Clin Endocrinol Metab. 2014; 99(11): E2335-E2340. 